\title{
Top-down MS for rapid methionine oxidation site assignment in filgrastim
}

\author{
Johann Holzmann • Anna Hausberger • \\ Alfred Rupprechter • Hansjoerg Toll
}

Received: 3 May 2013 / Revised: 7 June 2013 / Accepted: 10 June 2013 / Published online: 6 July 2013

(C) The Author(s) 2013. This article is published with open access at Springerlink.com

\begin{abstract}
Protein therapeutics have emerged as a major new class of pharmaceuticals. One important shelf-life-limiting factor of biopharmaceuticals is methionine oxidation, and therefore, it is important that analytical methods are able to thoroughly characterize all possible oxidized variants. Here, we present a fast and sensitive method to perform online methionine oxidation site assignment using granulocyte colony-stimulating factor (filgrastim) as a model. The method is based on top-down MS using the all-ion fragmentation mode of the Exactive benchtop mass spectrometer. Conditions that provide information on the intact mass of the protein as well as on fragment ions that allow unambiguous site assignment of methionine oxidation in filgrastim variants as low as $0.12 \%$ of total peak area in a chromatographic time scale were identified. Using this method, we performed methionine oxidation site assignment in $\mathrm{H}_{2} \mathrm{O}_{2}$-stressed filgrastim and in filgrastim which was stored at intended conditions, respectively. We show that the relative abundance of oxidation species observed in filgrastim stored under intended conditions differs strikingly from the oxidized species observed after $\mathrm{H}_{2} \mathrm{O}_{2}$ stress. Additionally, we report an oxidized filgrastim variant that has not been previously described in the literature.
\end{abstract}

Keywords G-CSF · Filgrastim · Mass spectrometry · All-ion fragmentation · Top-down MS · Methionine oxidation · Site assignment

J. Holzmann $(\bowtie) \cdot$ A. Hausberger $\cdot$ A. Rupprechter $\cdot$ H. Toll $(\bowtie)$

Sandoz GmbH, Biochemiestrasse 10, 6250 Kundl, Austria

e-mail: johann.holzmann@sandoz.com

e-mail: hansjoerg.toll@sandoz.com

\section{Introduction}

Granulocyte colony-stimulating factor (G-CSF) is a hematopoietic growth factor and cytokine that stimulates the production of neutrophils and affects neutrophil progenitor proliferation, differentiation, and cell functional activation [1, 2]. Recombinant human G-CSF (rhG-CSF; INN filgrastim) expressed in Escherichia coli was one of the first biopharmaceuticals to be commercialized (Neupogen ${ }^{\circledR}$; Amgen Inc.). It is a 175-amino acid long non-glycosylated polypeptide with an additional N-terminal methionine residue not found in the native endogenous human protein. Filgrastim is largely used to treat neutropenia as well as infectious complications, which can be associated with chemotherapy [3, 4]. Following the patent expiration of the reference product in the European Union, three biosimilar filgrastims have been approved [5], one of which is Zarzio ${ }^{\circledR}$ (Sandoz). Due to the introduction of more affordable biosimilar versions of filgrastim, some countries have managed to move filgrastim back to prophylactic use in order to reduce the incidence of febrile neutropenia after chemotherapy, thereby preventing hospital readmission due to infections [6-9]. Patient-friendly application devices together with new formulations are crucial to further enhance patient access. Their development, however, requires close monitoring of filgrastim-related impurities that occur due to physical or chemical degradation of the protein.

One important shelf-life-limiting degradation product of filgrastim is the oxidation of methionine residues to their sulfoxide derivatives. Filgrastim contains four methionine residues $\left(\mathrm{Met}^{1}, \mathrm{Met}^{122}\right.$, $\mathrm{Met}^{127}$, and $\mathrm{Met}^{138}$ ) and an extensive body of literature is available on the analytical and biological characterization of $\mathrm{H}_{2} \mathrm{O}_{2}$-induced oxidized variants [10-15]. Quantification of methionine oxidation in proteins is usually performed by reverse-phase HPLC (RP-HPLC). Since each of the four methionine residues in filgrastim is susceptible to oxidation, it is important that analytical methods are able to 
thoroughly characterize all possible oxidized variants. Assignment of the methionine oxidation site is routinely performed by isolation of individual oxidized species and subsequent enzymatic (e.g., GluC) or chemical (e.g., CNBr) cleavage of the protein (bottom-up approach). The resulting peptides are then subjected to liquid chromatography-mass spectrometry (LC-MS) analysis (Fig. 1).

Site assignment of methionine oxidation using this workflow has two major drawbacks: (1) a substantial amount of sample is required to allow variant fractionation and subsequent peptide mapping (especially for low abundant variants) and (2) the procedure is time consuming, due to elongated sample preparation and LC-MS analysis. Hence, there exists a need for a fast and sensitive method for oxidation site assignment in biopharmaceuticals such as filgrastim.

With the continuous improvement in instrumentation and data analysis software, top-down MS is becoming a highly attractive method for the characterization of proteins [16-21]. The Orbitrap family of MS analyzers is a popular and powerful tool for protein characterization due to its capabilities of high resolution and high mass accuracy. Top-down MS using this instrumentation has mostly been reported for proteins with molecular weights $<30 \mathrm{kDa}$, but has also been successfully implemented for larger proteins up to the size of intact antibodies $(\sim 150 \mathrm{kDa})$, albeit with lower resolution settings [22-27].

Herein, we report - for the first time - a top-down approach on a benchtop Exactive mass spectrometer for online methionine oxidation site assignment in a biopharmaceutical, using the microbially produced drug filgrastim. The method provides information on the intact mass of the protein variant as well as on fragment ions that allow unambiguous site assignment of methionine oxidation necessary for site-specific quantification of methionine oxidation via UV. Using this top-down approach, we also characterized an oxidized filgrastim variant that has not been previously reported in the literature.

\section{Materials and methods}

Chemicals and study material

All chemicals purchased were of highest purity available. Trifluoroacetic acid was obtained from Sigma-Aldrich (Steinheim, Germany). HPLC grade water and acetonitrile were purchased from J.T. Baker (Deventer, The Netherlands). Forced oxidation using $\mathrm{H}_{2} \mathrm{O}_{2}$ was performed on Sandoz filgrastim drug product $\left(\mathrm{Zarzio}^{\circledR}\right.$, formulation $\mathrm{A}$ ) produced in
Fig. 1 The classic approach for the assignment of methionine oxidation involves isolation of the individual oxidized species and subsequent peptide mapping using a specific protease (e.g., GluC). (a) Chromatographic separation of oxidized filgrastim variants following $\mathrm{H}_{2} \mathrm{O}_{2}$ treatment. (b) Peptide mapping of $\mathrm{Ox}-2$ and Ox-3 fractions demonstrating unique oxidation peak whose site assignment could be elucidated using MS and MS/ MS analysis (peaks labeled according to theoretical digest, e.g., G1 represents the first peptide of a GluC digest; mass spectrometry data are not shown) a

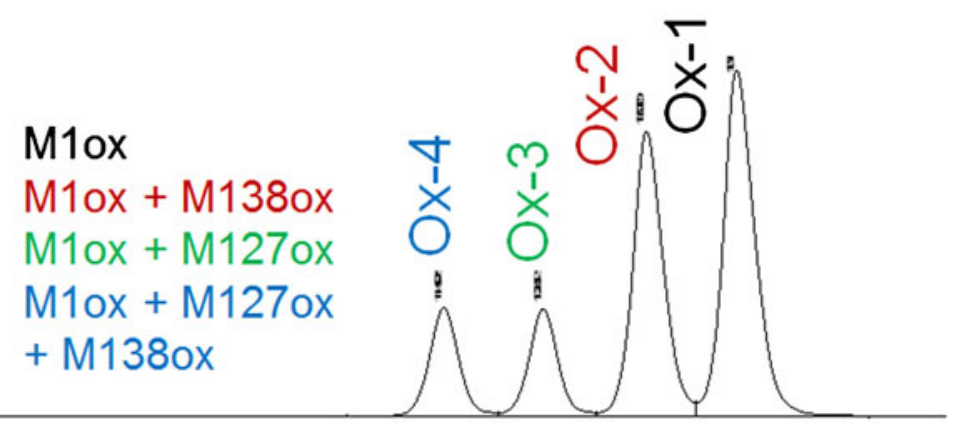

b

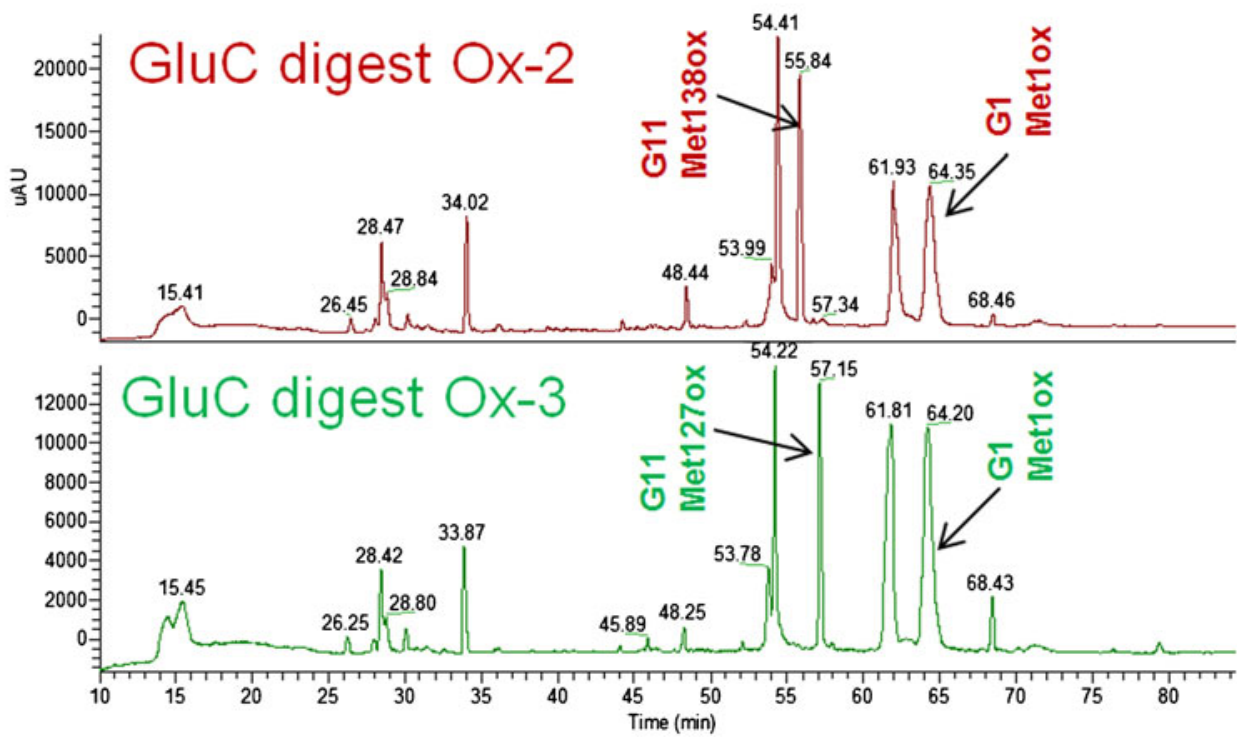


house; the same batch of Zarzio ${ }^{\circledR}$ was used for selection of the diagnostic fragment ions (Figs. 2, 3, and 4). For online Met oxidation site assignment in samples stored under intended conditions (Fig. 5), Sandoz filgrastim development samples (formulation B) and Neupogen ${ }^{\circledR}$ were used. Neupogen ${ }^{\circledR}$ was sourced from Amgen (Amgen Inc., Thousand Oaks, CA) and stored under intended storage conditions.

\section{Direct infusion of filgrastim and MS analysis}

Sandoz filgrastim drug product (Zarzio ${ }^{\circledR}$, formulation A) was buffer exchanged into water using Microcon YM-3 centrifugal filter devices (Millipore, Bedford, MA) and diluted with a solution of $50 \%$ acetonitrile $(\mathrm{ACN})$ in $0.1 \%$ trifluoroacetic acid (TFA) to a final concentration of $1.1 \mathrm{mg} / \mathrm{ml}$. A $500-\mu \mathrm{l}$ Hamilton syringe was used to infuse the sample directly into the Exactive mass spectrometer at a flow rate of $10 \mu 1 / \mathrm{min}$. The Exactive MS was operated in an all-ion fragmentation (AIF) mode with the following settings applied: spray voltage $4 \mathrm{kV}$, capillary temperature $275^{\circ} \mathrm{C}$, sheath gas 20 , aux gas 8 , scan range 200-2,000 m/z, resolution ultra high, AGC target 1e6, max inject time $100 \mathrm{~ms}$, and microscans 10 . Higher energy collision dissociation (HCD) fragmentation energy was manually varied between 15 and $45 \mathrm{eV}$. For data evaluation, spectra acquired within a time frame of approximately $10 \mathrm{~s}$ were averaged and deconvoluted using the Xtract algorithm implemented into Xaclibur 2.1 Software (Thermo Scientific, Bremen, Germany). The following parameters were used for deconvolution: $S / N$ threshold 2, fit factor $44 \%$, remainder
$25 \%$, and max charge 20 . The assignment of deconvoluted AIF spectra was done manually using GPMAW 9.02 software (Lighthouse data, Odense, Denmark) to predict the masses of the individual fragment ions.

HPLC separation and all-ion fragmentation on Exactive MS

Nine micrograms of Sandoz filgrastim development sample (drug product, formulation B), Sandoz filgrastim drug product (Zarzio ${ }^{\circledR}$; formulation A), and Neupogen ${ }^{\circledR}$, respectively, was separated on a Zorbax 300 SB-C18 column $(4.6 \times 150 \mathrm{~mm}$, $3.5 \mu \mathrm{m}$ particle size $)$ with a gradient of solutions A $(0.1 \%$ TFA in water) and $\mathrm{B}(0.1 \% \mathrm{TFA}$ in $\mathrm{ACN})$ at a flow rate of $1 \mathrm{ml} / \mathrm{min}$ : $25 \mathrm{~min}$ from $25 \% \mathrm{~B}$ to $54 \% \mathrm{~B}$ followed by a $32-\mathrm{min}$ gradient from $54 \%$ B to $73 \%$ B. After UV and fluorescence detection, the flow was split 1:5 and then electrosprayed into the Exactive MS. The mass spectrometer was again operated in the AIF mode with settings applied as discussed above, except that the mass range was set to 300 to $2,200 \mathrm{~m} / \mathrm{z}$. Deconvolution and data interpretation were performed as described above.

\section{Results}

Selection of diagnostic fragment ions

Direct infusion experiments using desalted filgrastim were carried out for the selection of diagnostic fragment ions and the optimization of HCD settings. Figure 2 shows the sequence

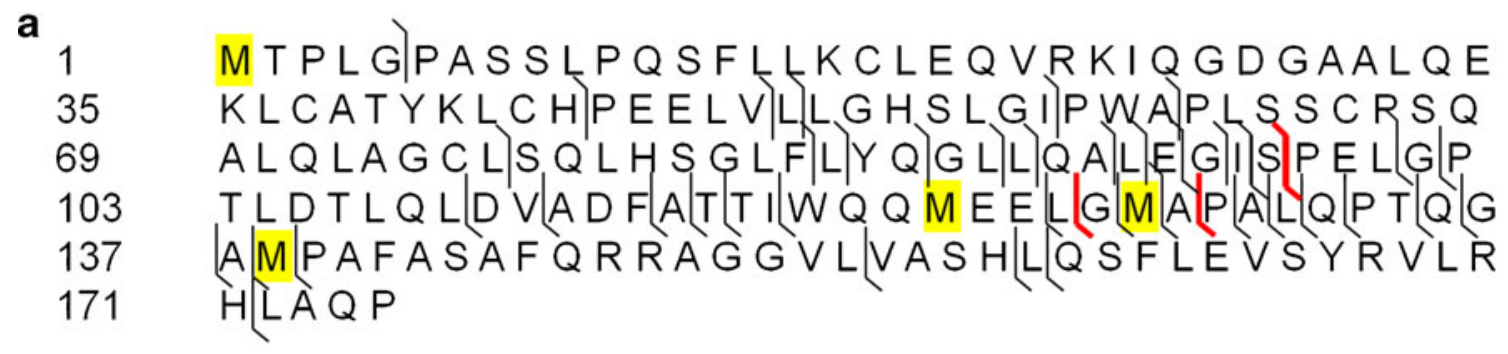

b

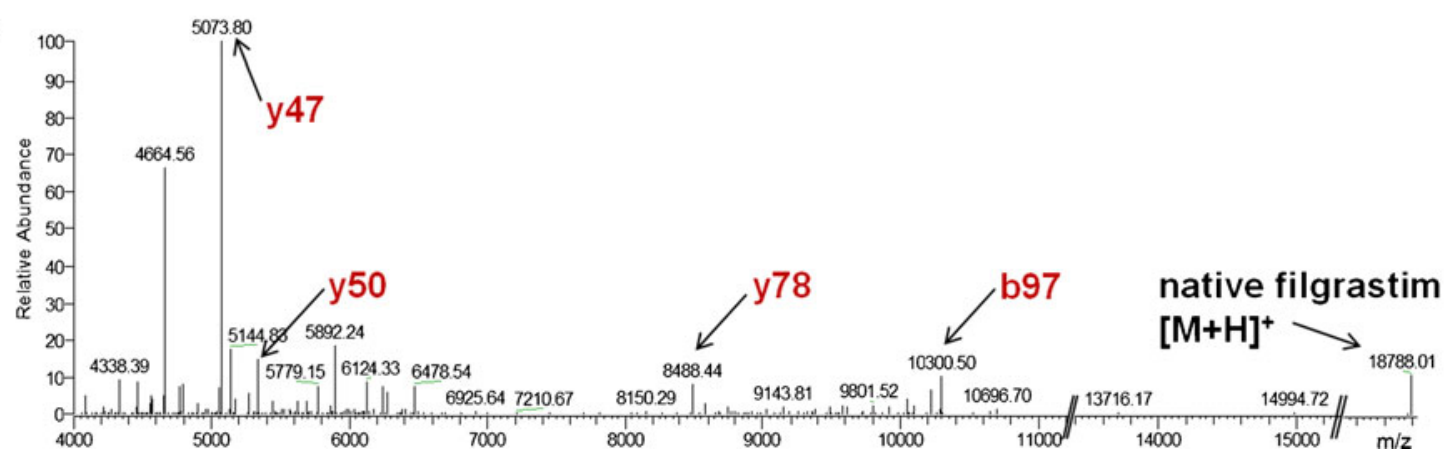

Fig. 2 Sandoz filgrastim drug product $\left(\right.$ Zarzio $\left.^{\circledR}\right)$ is a biosimilar of filgrastim (recombinant human granulocyte colony-stimulation factor (rhG-CSF)) and has a molecular weight of $18.8 \mathrm{kDa}$. It contains four Met residues at positions 1, 122, 127, and 138 (highlighted in yellow). (a)

Sequence coverage generated by HCD with $35 \mathrm{eV}$. (b) Deconvoluted HCD spectra of Sandoz filgrastim drug product $\left(\right.$ Zarzio $\left.^{\circledR}\right)$. The fragment ions y47, y50, y78, and b97 allow assignment of all four Met residues (highlighted in red), which can be oxidized under stressed conditions 
Fig. 3 Sandoz filgrastim drug product $\left(\right.$ Zarzio $\left.^{\circledR}\right)$, a biosimilar filgrastim, was subjected to oxidation with (a) $0.1 \%$ and (b) $0.5 \%$ final concentration of

$\mathrm{H}_{2} \mathrm{O}_{2}$, separated on a RP C18 column and electrosprayed into an Exactive mass spectrometer. Peak assignment was performed by detection of diagnostic ions following HCD fragmentation at $35 \mathrm{eV}$. Peaks labeled in brackets indicate missing fragment ions due to low abundance. The HCD spectra of peaks labeled in red are shown in Fig. 4. The site of Met oxidation can be unambiguously assigned in peaks as low as $0.70 \%$ of total peak area (M138ox+M127ox)

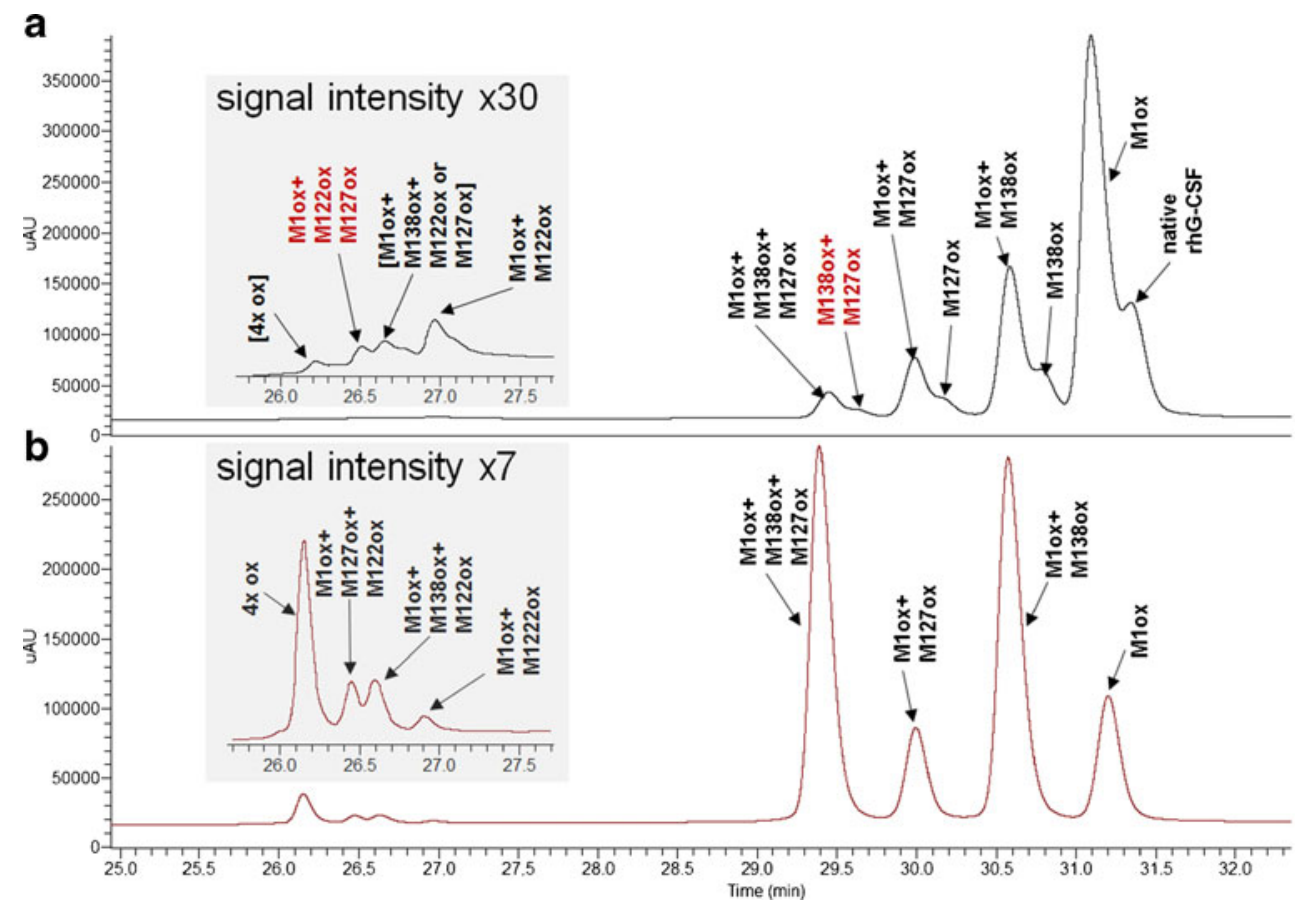

coverage and representative HCD spectrum obtained using our top-down analysis of Sandoz filgrastim drug product (Zarzio ${ }^{\circledR}$ ), a biosimilar filgrastim, stored under intended storage conditions.
Despite sequence coverage of only $25 \%$, all-ion fragmentation in the C-trap of the Exactive instrument produced fragment ions (highlighted in red in Fig. 2a) that allowed

a

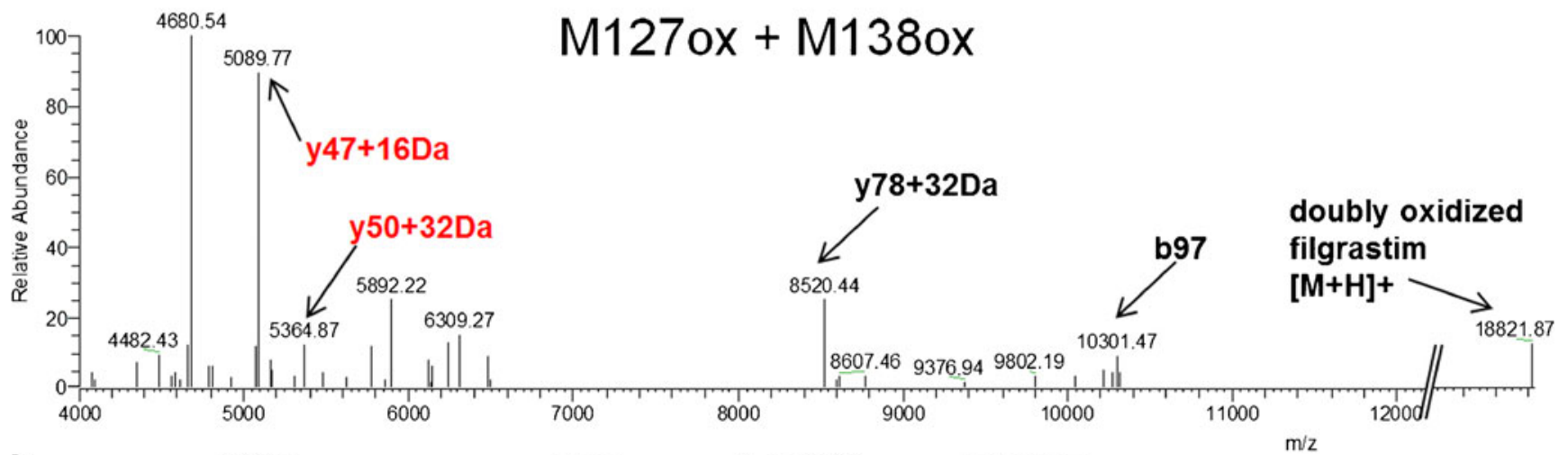

b $_{100}{ }_{466454}^{5073.78} \quad$ M1ox + M122ox+ M127ox

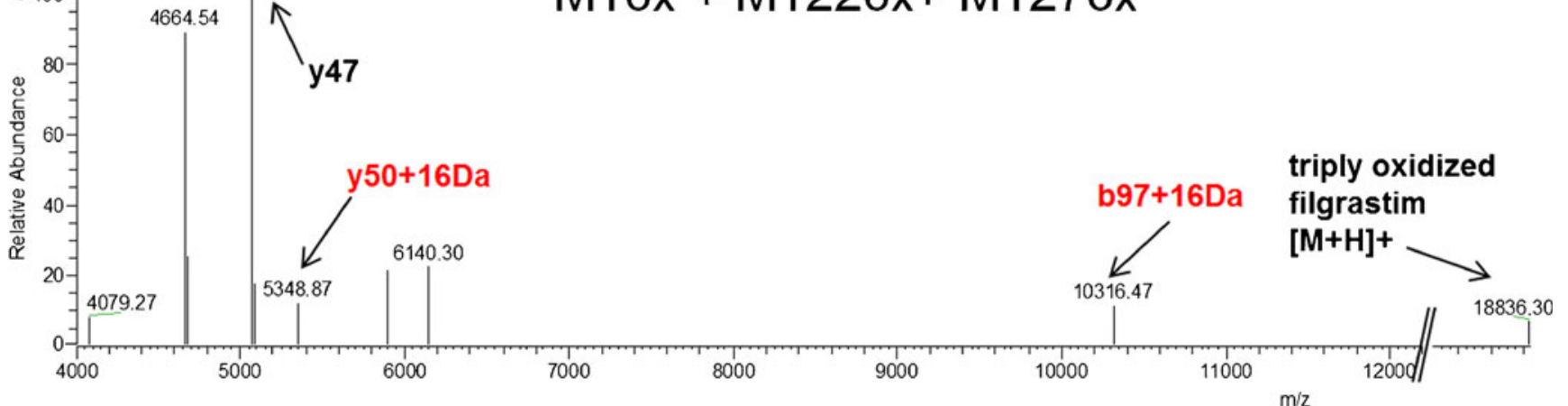

Fig. 4 HCD spectra of low abundance oxidized filgrastim variants labeled in red in Fig. 3. (a) The diagnostic fragment ions for M127ox + M138ox $(\mathrm{y} 47+16 \mathrm{Da}$ and y50+32 Da) were unambiguously identified in the HCD spectra despite representing only $0.7 \%$ of the total peak area. (b) Due to the low abundance of the triply oxidized variant, which represents $0.12 \%$ of the total peak area, the diagnostic fragment ion y78 is missing. However, the combination of native mass $(18,788.01+48.3)$ and diagnostic ions y47, $\mathrm{y} 50+16 \mathrm{Da}$, and b97+16 Da allows the sites of oxidation to be elucidated 


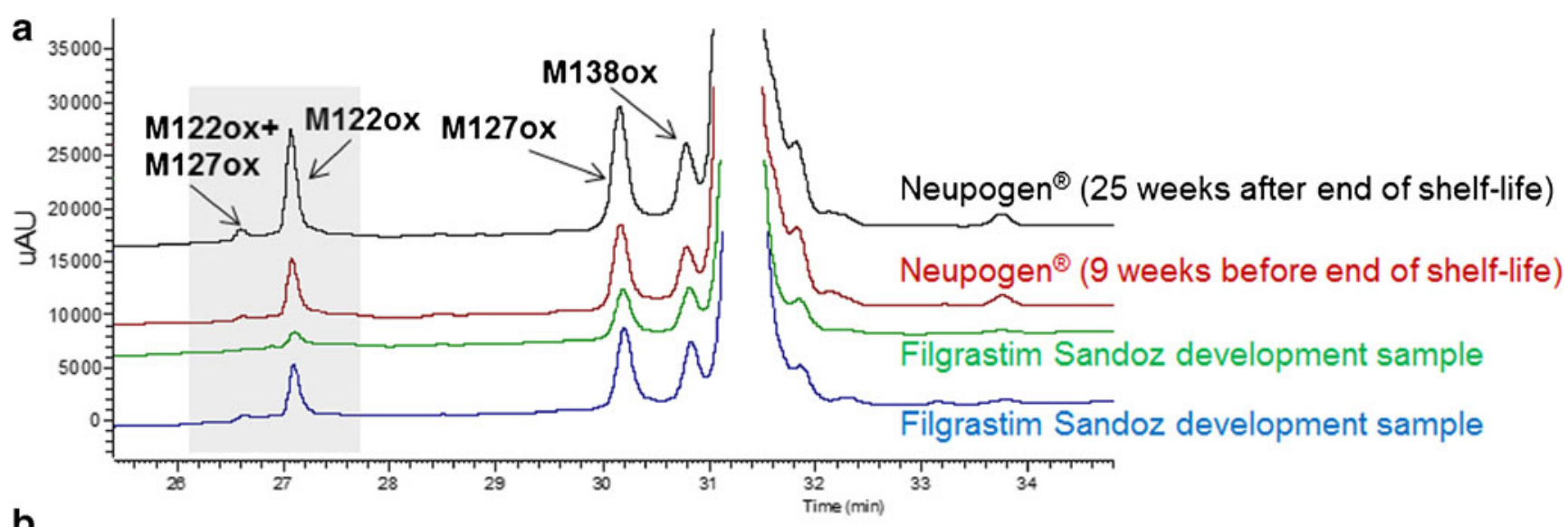

b
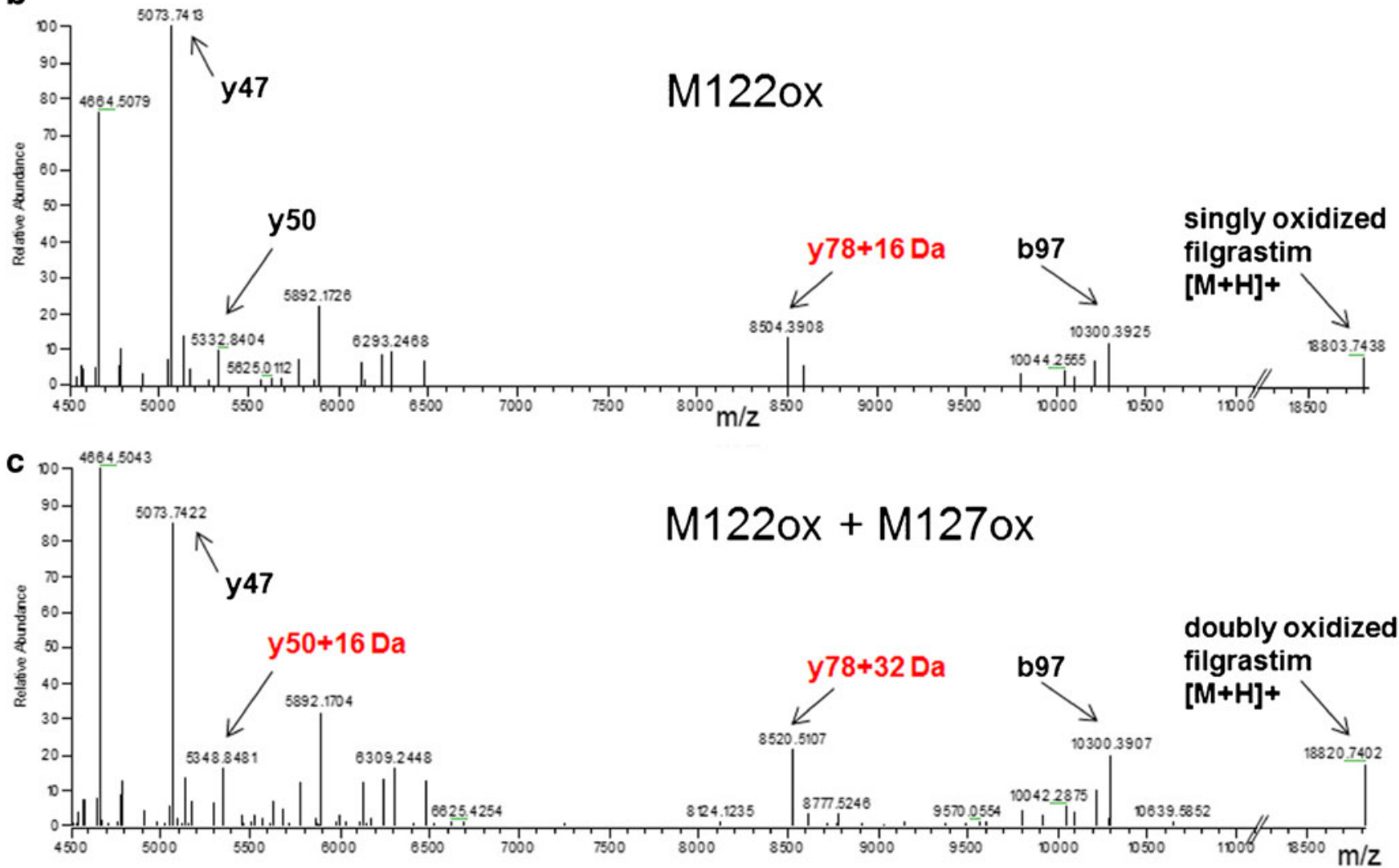

Fig. 5 (a) UV chromatogram of Neupogen ${ }^{\circledR}$ (black trace; analysis performed 25 weeks after end-of-shelf-life, red trace analysis performed 9 weeks before end-of-shelf-life, stored at $5 \pm 3{ }^{\circ} \mathrm{C}$ ) and

the discrimination of all four oxidized methionine residues found in filgrastim with reasonable intensity (Fig. 2b). Fragment ion y47 (theoretical mass of 5,073.71 Da) is the most abundant ion found in the HCD spectrum and is diagnostic for $\mathrm{Met}^{138}$. Fragment ion y50 (theoretical mass of 5,332.81 Da) contains $\mathrm{Met}^{127}$ and Met ${ }^{138}$, which, after considering the mass of $y 47$, is diagnostic for Met ${ }^{127}$. Similarly, fragment ion y 78 (theoretical mass of $8,488.35 \mathrm{Da}$ ) contains three methionine residues, $\mathrm{Met}^{122}$, Met ${ }^{127}$, and $\mathrm{Met}^{138}$, but becomes diagnostic for $\mathrm{Met}^{122}$ af0ter considering the masses of y50 and y47. Finally, fragment ion b97 (theoretical mass filgrastim Sandoz development samples (green and blue traces, stored for 34 weeks at $5 \pm 3{ }^{\circ} \mathrm{C}$ ). (b) $\mathrm{HCD}$ spectrum of variant peak eluting at $27.2 \mathrm{~min}$ and (c) HCD spectrum of variant eluting at $26.8 \mathrm{~min}$

of $10,300.34 \mathrm{Da}$ ) is diagnostic for Met ${ }^{1}$. In addition to the diagnostic ions, we also observed some unfragmented parent ion which allowed the simultaneous measurement of the intact mass of filgrastim (theoretical monoisotopic mass $[\mathrm{M}+\mathrm{H}]^{+}$of $\left.18,787.68 \mathrm{Da}\right)$. The average mass deviation over all measured fragment ions was $11 \mathrm{ppm}$. As an alternative approach, we also tested in-source collision-induced dissociation, but were not able to detect fragment ions that could discriminate between methionines at positions 127 and 138 (data not shown). From Fig. 1b, it can be deduced that the sensitivity of the HCD method is limited by the intensity of 
fragments y50, y78, and b97. To optimize the generation of these limiting ions, filgrastim was subjected to higher energy collision dissociation using different acceleration potentials. By testing increasing collision potentials, fragmentation with $35 \mathrm{eV}$ was selected as the best compromise between decreasing b97 intensity and increasing y78 intensity (Table 1).

Online Met oxidation site assignment in $\mathrm{H}_{2} \mathrm{O}_{2}$-stressed filgrastim

To simulate oxidative stress conditions, Sandoz filgrastim drug product $\left(\mathrm{Zarzio}^{\circledR}\right.$ ) was treated with 0.1 and $0.5 \% \mathrm{H}_{2} \mathrm{O}_{2}$, respectively, final concentration of $\mathrm{H}_{2} \mathrm{O}_{2}$. Oxidized filgrastim variants were separated by RP-HPLC and online methionine oxidation site assignment was performed using our top-down method on a benchtop Exactive MS. Oxidation of filgrastim under these conditions produced a number of oxidized variants that were separated by RP-HPLC (Fig. 3).

Oxidation site assignment using our top-down approach was possible for all detected oxidized filgrastim variants. Figure 4 shows two examples of HCD spectra used for the site assignment of methionine oxidation (variants labeled in red in Fig. 3a).

In Fig. 4a, the HCD spectrum of variant denoted as $\operatorname{Met}^{1380 x}+\operatorname{Met}^{1270 x}\left(0.1 \% \mathrm{H}_{2} \mathrm{O}_{2}\right.$ stress $)$ is shown. Despite the fact that this low abundance variant represents only $0.70 \%$ of total peak area (see Fig. 3a), all diagnostic fragment ions as well as the intact mass of the protein were detected, albeit with higher mass errors for the low intensity fragments. For example, while the high-intensity fragment ion $y 47+16 \mathrm{Da}$ exhibited a mass error of $13 \mathrm{ppm}$, the measured mass of the intact protein deviated by $2.1 \mathrm{Da}$ from its theoretical mass. This high mass deviation for the intact protein could be explained by an isotope shift introduced by the deconvolution software due to low signal intensity. In conclusion, the data show that this filgrastim variant is doubly oxidized at residues $\mathrm{Met}^{127}$ and $\mathrm{Met}^{138}$. Figure $4 \mathrm{~b}$ shows the HCD spectrum of the variant denoted as $\mathrm{Met}^{1 \mathrm{10x}}+\mathrm{Met}^{122 \mathrm{ox}}+\operatorname{Met}^{1270 x}\left(0.1 \% \mathrm{H}_{2} \mathrm{O}_{2}\right.$, labeled in red in Fig. 3a). Due to the low abundance of this variant which represents only $0.12 \%$ of total peak area, the diagnostic ion y $78+32 \mathrm{Da}$ is missing. However, by considering the parent ion mass measurement, which is 48.3 Da heavier than the non-oxidized filgrastim parent ion, together with the diagnostic ions y47, y50+16 Da, and b97+16 Da allowed us to conclude that this filgrastim variant is triply oxidized at residues $\mathrm{Met}^{1}, \mathrm{Met}^{122}$, and $\mathrm{Met}^{127}$. For two very low abundant impurities observed in $0.1 \% \mathrm{H}_{2} \mathrm{O}_{2}$ stress, the native mass of the variant denoted with $\left[\mathrm{Met}^{1 \mathrm{Ox}}+\mathrm{Met}^{138 \mathrm{ox}}+\mathrm{Met}^{122 \mathrm{ox}}\right.$ or $\mathrm{Met}^{1270 x}$ ] was in agreement with triply oxidized filgrastim; however, fragment ions representing the oxidation state at y50 and y78 were missing, thus impairing position assignment. The abundance of the variant labeled with $[4 \times \mathrm{ox}]$ in the $0.1 \%$ $\mathrm{H}_{2} \mathrm{O}_{2}$ sample was too low to allow charge determination in the HCD spectrum and subsequent deconvolution of the tenfoldcharged parent ion (theoretical average mass of native filgrastim is $1,880.89 \mathrm{Th}$ in the tenfold charge state). However, a signal was detected at $1,187.30 \mathrm{Th}$, which is close to the mass of the tenfold-charged parent ion $+4 \times 16 \mathrm{Da}$, suggesting the presence of filgrastim with all four methionine residues oxidized (not shown). Unambiguous oxidation site assignment of $4 \times$ oxidized filgrastim was possible in $0.5 \%$ $\mathrm{H}_{2} \mathrm{O}_{2}$-stressed sample where these variants were more abundant (data not shown). While its implications remain unclear, it is interesting to note that in the $\mathrm{H}_{2} \mathrm{O}_{2}$-stressed samples, oxidation at $\mathrm{Met}^{122}$ was only observed in combination with oxidation at other methionine residues. Noteworthy, oxidation of $\mathrm{Met}^{122}$ resulted in a major shift in RP-HPLC retention time.

Isolated $\mathrm{Met}^{122}$ oxidation in biosimilar and originator filgrastim stored under intended conditions

In the process of characterizing new formulations of filgrastim being developed (Sandoz filgrastim development sample, formulation B), two new variants were observed. These new variants eluted much earlier in RP-HPLC (Fig. 5a), suggesting oxidation at Met ${ }^{122}$ alone or in combination with other methionine residues. Fragment ion analysis was able to confirm that the identity of the major unique peak is in fact a filgrastim variant with a singly oxidized Met $^{122}$ residue (Fig. 5b). In addition, we identified a very low abundant variant as $\mathrm{Met}^{122}$ plus Met ${ }^{127}$ oxidation.

Isolated Met ${ }^{122}$ has been reported by Reubsaet et al. [12] in $\mathrm{H}_{2} \mathrm{O}_{2}$-stressed samples. However, data on G-CSF oxidation behavior published more recently as well as our data strongly
Table 1 For optimization of HCD, Sandoz filgrastim drug product $\left(\right.$ Zarzio $^{\mathbb{R}}$ ) was subjected to fragmentation in the C-trap using different acceleration potentials. Listed are intensities of selected fragment ions relative to the highest signal in the spectrum (y47). $35 \mathrm{eV}$ was selected as the best compromise between decreasing b97 intensity and increasing y78 intensity upon increase of acceleration potential

\begin{tabular}{lllllll}
\hline Fragment ion & Mass [Da] & Diagnostic for & HCD 22 eV & HCD 28 eV & HCD 35 eV & HCD 40 eV \\
\hline b97 & $10,300.3$ & Met1 & 5 & 5 & 4 & 1 \\
y78 & $8,488.4$ & Met122 (considering y50 and y47) & 0 & 3 & 5 & 5 \\
y50 & $5,332.8$ & Met127 (considering y47) & 18 & 20 & 20 & 100 \\
y47 & $5,073.7$ & Met138 & 100 & 100 & 100 \\
\hline
\end{tabular}


suggest that the oxidized variant denoted as oxidized Met $^{122}$ by Reubsaet and colleagues is actually oxidized Met ${ }^{1}$.

Therefore, this is the first report of a filgrastim variant with an isolated oxidation of Met ${ }^{122}$. Noteworthy, both variants were detected in filgrastim Sandoz development samples (Fig. 5, green and blue traces) as well as in Neupogen ${ }^{\circledR}$ (Fig. 5a, black and red traces).

\section{Discussion}

In filgrastim, oxidation of methionine residues to their sulfoxide derivatives is an important degradation pathway and might become the shelf-life-limiting factor. Therefore, it is necessary to thoroughly characterize oxidized filgrastim variants. Oxidation of a particular methionine residue can depend on its solvent accessibility. While $\operatorname{Met}^{1}, \operatorname{Met}^{138}$, and $\mathrm{Met}^{127}$ can interact with solvent molecules, Met ${ }^{122}$ is located at a hydrophobic region of the protein [28-30]. Oxidation of $\mathrm{Met}^{122}$ was described as relatively slow and affected by the oxidation of $\mathrm{Met}^{127}$. The oxidation of $\mathrm{Met}^{122}$ independent from the oxidation of $\mathrm{Met}^{127}$ was confirmed in filgrastim mutants where $\mathrm{Met}^{127}$ was substituted for a leucine residue (M127L variant), although with very slow oxidation rates [10].

In agreement with structural data and with earlier reports, this study demonstrates $\mathrm{Met}^{1}$ to be most susceptible to $\mathrm{H}_{2} \mathrm{O}_{2}$ induced oxidation, followed by $\mathrm{Met}^{138}$ and $\mathrm{Met}^{127}[10,11$, 28-31]. In $\mathrm{H}_{2} \mathrm{O}_{2}$-stressed samples, oxidation of $\mathrm{Met}^{122}$ accounted for only a minor fraction of the detected impurities (slow oxidation rate) and was always associated with the oxidation of at least another methionine residue. Interestingly, single oxidation at $\mathrm{Met}^{122}$ was observed in our filgrastim biosimilar development samples as well as in originator batches of Neupogen ${ }^{\circledR}$ stored at indicated conditions. As shown in Fig. 5a, the degree of single oxidation of Met $^{122}$ in these samples was comparable to the amount of single oxidation at $\mathrm{Met}^{127}$ or $\mathrm{Met}^{138}$ and approximately tenfold more abundant than filgrastim with dual oxidation at both $\mathrm{Met}^{122}$ and $\mathrm{Met}^{127}$. Although the kinetics of these oxidation events has not been characterized in this study, the steady-state oxidation species observed in filgrastim stored under clinically relevant conditions differs strikingly from the kinetics reported for $\mathrm{H}_{2} \mathrm{O}_{2}$ induced oxidation, which follows the order $\mathrm{Met}^{1}>\mathrm{Met}^{138}>$ $\operatorname{Met}^{127}>\operatorname{Met}^{122}[10,11,32]$. Therefore, $\mathrm{H}_{2} \mathrm{O}_{2}$ stress does not fully reflect the oxidative mechanisms which dominate during storage. Met ${ }^{122}$ is located at the hydrophobic core of filgrastim and its oxidation could induce a conformational change, possibly explaining the strong shift observed in retention time [28-30]. Interestingly, despite the likelihood of conformational changes associated with Met ${ }^{122}$ oxidation, previous studies in which Met ${ }^{122}$ was specifically oxidized using filgrastim mutants demonstrated that $\mathrm{Met}^{122}$ oxidation has only a minor effect on the biological activity [10]. However, given the fact that the rate of $\mathrm{Met}^{122}$ oxidation depends on the formulation buffer, oxidation of Met ${ }^{122}$ should be closely monitored, especially when aiming to develop a new buffer system [14, $15]$.

Rapid site assignment of this new oxidized filgrastim variant was possible with the use of a novel top-down approach using a benchtop mass spectrometer. This Exactive instrument is a recently introduced stand-alone Orbitrap analyzer and offers top-down capabilities using AIF in the instrument HCD cell, allowing high resolution and mass accuracy in both the full scan and AIF mode in a robust and economical format [32]. AIF does not allow the targeted selection of a specific precursor ion; therefore, it requires reasonably pure proteins for analysis. While this is certainly a problem in complex samples (e.g., periplasmic fractions), the impurities detected in the final product are sufficiently separated by RP-HPLC before MS detection. In fact, the AIF might lead to a higher sensitivity because parent ions from all charge states are subjected to fragmentation, while in "true" MS/MS approaches, only parent ions from a single charge state are selected for dissociation, limiting the detection capability. However, future experiments will have to be carried out to determine actual differences in method sensitivity. Based on our data, the limit of oxidation site assignment from RP-HPLC-separated filgrastim variants lies between $0.12 \%$ (Fig. 4b) and $0.70 \%$ (Fig. $4 a$ ) of total peak area. Compared to traditional isolation/bottom-up approaches, the high sensitivity of our top-down method translates into significantly lower sample requirements for characterization $(10 \mu \mathrm{g}$ per analysis for top-down compared to $2 \mathrm{mg}$ for bottom-up, assuming a variant of $\sim 1 \%$ abundance). Furthermore, because our method allows direct online analysis of RP-HPLC eluents, the time required for characterization was significantly shortened from about 1 week (for isolation, digestion, and MS analysis) to $1 \mathrm{~h}$.

\section{Conclusion}

The top-down approach outlined in this study describes a highly attractive alternative to the traditional approach of isolation/bottom-up analysis for methionine oxidation site assignment in biopharmaceuticals. With a demonstrated sensitivity as low as $0.12 \%$ of total peak area and a throughput of about one sample per hour, the described method is highly suitable for a thorough characterization of oxidized methionine residues in, e.g., formulation development. With respect to instrumentation, this work shows that the Exactive benchtop mass spectrometer operated in all-ion fragmentation mode is a sensitive and economic tool for online top-down characterization of less complex samples such as microbial derived biopharmaceuticals and their degradation products. 
Acknowledgments We would like to thank Jan Visser, Martin Schiestl, and William Lamanna from Sandoz Biopharmaceuticals for their thorough review and support.

Open Access This article is distributed under the terms of the Creative Commons Attribution License which permits any use, distribution, and reproduction in any medium, provided the original author(s) and the source are credited.

\section{References}

1. Metcalf D (1985) Cell 43:5-6

2. Nemunaitis J (1997) Drugs 54:709-729

3. Aapro MS, Bohlius J, Cameron DA, Dal LL, Donnelly JP, Kearney N, Lyman GH, Pettengell R, Tjan-Heijnen VC, Walewski J, Weber DC, Zielinski C (2011) Eur J Cancer 47:8-32

4. Dale DC (2002) Drugs 62(1):1-15

5. Gascon P (2012) Target Oncol 7(1):S29-S34

6. Sorgel F, Lerch H, Lauber T (2010) BioDrugs 24:347-357

7. Aapro M, Cornes P, Abraham I (2012) J Oncol Pharm Pract 18:171-179

8. Salesi N, Di CB, Colonna M, Veltri E (2012) Future Oncol 8:625-630

9. McCamish M, Woollett G (2012) Clin Pharmacol Ther 91:405-417

10. Lu HS, Fausset PR, Narhi LO, Horan T, Shinagawa K, Shimamoto G, Boone TC (1999) Arch Biochem Biophys 362:1-11

11. Pan B, Abel J, Ricci MS, Brems DN, Wang DI, Trout BL (2006) Biochemistry 45:15430-15443

12. Reubsaet JL, Beijnen JH, Bult A, Hop E, Scholten SD, Teeuwsen J, Underberg WJ (1998) J Pharm Biomed Anal 17:283-289

13. Skrlin A, Kosor KE, Gosak D, Prester B, Mrsa V, Vuletic M, Runac D (2010) J Pharm Biomed Anal 53:262-268

14. Yin J, Chu JW, Ricci MS, Brems DN, Wang DI, Trout BL (2004) Pharm Res 21:2377-2383
15. Yin J, Chu JW, Ricci MS, Brems DN, Wang DI, Trout BL (2005) Pharm Res 22:141-147

16. Kellie JF, Tran JC, Lee JE, Ahlf DR, Thomas HM, Ntai I, Catherman AD, Durbin KR, Zamdborg L, Vellaichamy A, Thomas PM, Kelleher NL (2010) Mol Biosyst 6:1532-1539

17. Macht M (2009) Bioanalysis 1:1131-1148

18. Zhou H, Ning Z, Starr AE, Abu-Farha M, Figeys D (2012) Anal Chem 84:720-734

19. Chen G, Warrack BM, Goodenough AK, Wei H, Wang-Iverson DB, Tymiak AA (2011) Drug Discov Today 16:58-64

20. Reid GE, McLuckey SA (2002) J Mass Spectrom 37:663-675

21. Breuker K, Jin M, Han X, Jiang H, McLafferty FW (2008) J Am Soc Mass Spectrom 19:1045-1053

22. Tran JC, Zamdborg L, Ahlf DR, Lee JE, Catherman AD, Durbin KR, Tipton JD, Vellaichamy A, Kellie JF, Li M, Wu C, Sweet SM, Early BP, Siuti N, LeDuc RD, Compton PD, Thomas PM, Kelleher NL (2011) Nature 480:254-258

23. Mazur MT, Seipert RS, Mahon D, Zhou Q, Liu T (2012) AAPS J 14:530-541

24. Zhang Z, Shah B (2007) Anal Chem 79:5723-5729

25. Zhang J, Liu H, Katta V (2010) J Mass Spectrom 45:112-120

26. Bondarenko PV, Second TP, Zabrouskov V, Makarov AA, Zhang Z (2009) J Am Soc Mass Spectrom 20:1415-1424

27. Macek B, Waanders LF, Olsen JV, Mann M (2006) Mol Cell Proteomics 5:949-958

28. Hill CP, Osslund TD, Eisenberg D (1993) Proc Natl Acad Sci U S A 90:5167-5171

29. Werner JM, Breeze AL, Kara B, Rosenbrock G, Boyd J, Soffe N, Campbell ID (1994) Biochemistry 33:7184-7192

30. Aritomi M, Kunishima N, Okamoto T, Kuroki R, Ota Y, Morikawa K (1999) Nature 401:713-717

31. Chu JW, Yin J, Wang DI, Trout BL (2004) Biochemistry 43:10191029

32. Geiger T, Cox J, Mann M (2010) Mol Cell Proteomics 9:22522261 